\title{
Flora vascular de um inselbergue no estado da Paraíba, Nordeste do Brasil
}

\author{
Elisabeth Emília Augusta Dantas Tölke ${ }^{1}$ \\ Joan Bruno Silva ${ }^{2}$ \\ Acácia Renally Lopes Pereira ${ }^{2}$ \\ José Iranildo Miranda de Melo ${ }^{2 *}$ \\ ${ }^{1}$ Programa de Pós-Graduação em Biologia Vegetal, Instituto de Biologia \\ Universidade Estadual de Campinas, CEP 13083-970, Campinas - SP, Brasil \\ ${ }^{2}$ Universidade Estadual da Paraíba, Centro de Ciências Biológicas e da Saúde \\ Departamento de Biologia, CEP 58429-500, Campina Grande - PB, Brasil \\ *Autor para correspondência \\ jimmelo@pq.cnpq.br
}

\section{Resumo}

Este trabalho caracterizou a composição florística de um inselbergue no município de Puxinanã, estado da Paraíba, Nordeste do Brasil. Foram registradas 97 espécies distribuídas em 35 famílias. Asteraceae (12 spp.), Euphorbiaceae e Fabaceae (nove spp. cada), Rubiaceae e Convolvulaceae (oito spp. cada) foram as cinco famílias melhor representadas em número de espécies, englobando 47,42\% do total das registradas. O hábito herbáceo foi o predominante estando presente em 39\% das espécies. Em seguida, sobressaíram-se as espécies arbustivas (22\%), trepadeiras (20\%), e com um número menor de representantes os hábitos rupícola (6\%), subarbustivo (5\%), arbóreo (4\%) e epífito (1\%). Passiflora luetzelburgii Harms (Passifloraceae), constitui nova referência para a flora do estado. Os dados obtidos fornecem subsídios para implementação de possíveis ações conservacionistas e o uso racional da área estudada e para a flora e vegetação da Caatinga.

Palavras-chave: Caatinga, Florística, Puxinanã, Semiárido

\section{Abstract}

Vascular flora of an inselberg in the state of Paraíba, in Northeast Brazil. This study characterized the floristic composition of an inselberg in the Puxinanã municipality, of Paraíba, in Northeast Brazil. Ninety-seven species were recoded that belong to 35 families: Asteraceae (12 spp.), Euphorbiaceae and Fabaceae (nine spp. each), Rubiaceae and Convolvulaceae (eight spp. each) were the richest families, comprising $47.42 \%$ of the total species registered. The herbaceous habit was predominant, occurring in $39 \%$ of the species, followed by species of shrubs (22\%), vines (20\%), rupicolous plants $(6 \%)$, subshrubs $(5 \%)$, trees $(4 \%)$ and epiphytes $(1 \%)$. Passiflora luetzelburgii Harms (Passifloraceae) is a new record for the flora of the state. The data from this study provides information that could be used in the conservation and rational use of the study area, and contributes to what is known about the flora and vegetation of the Caatinga.

Key words: Caatinga, Floristics, Puxinanã, Semi-arid 


\section{Introdução}

Inselbergues são montanhas ou grupos de montanhas monolíticas solitárias que emergem abruptamente acima da planície que os cercam (BARTHLOTT et al., 1993; POREMBSKI et al., 1997; BURKE, 2002; POREMBSKI, 2007). O termo inselbergue foi introduzido em 1900 pelo geólogo Bornhardt para designar afloramentos rochosos que consistem de granitos e gnaisses de origem pré-cambriana (BARTHLOTT et al., 1993; BURKE, 2002). Ocorrem principalmente em regiões tropicais e subtropicais, sendo mais raros em regiões temperadas e na América do Norte (BARTHLOTT et al., 1993; POREMBSKI, 2007).

Caracterizam-se por apresentarem condições microclimáticas e edáficas particulares, como, por exemplo, temperaturas mais elevadas em relação ao entorno, retenção de calor, retenção de água formando poças isoladas, ação acentuada dos ventos, entre outras (BARTHLOTT et al., 1993; POREMBSKI, 2007; ARAÚJO et al., 2008) e, em regiões tropicais, se comportam como ilhas xéricas em ambientes úmidos, enquanto em regiões desérticas constituem um refúgio para diversas espécies vegetais (BURKE, 2002).

De acordo com Oliveira e Godoy (2007) as plantas crescem diretamente sobre a rocha ou em ilhas de vegetação, gerando uma paisagem que varia segundo a declividade da rocha e a profundidade do substrato. No bioma Caatinga a flora dos inselbergues se encontra influenciada por filtros ambientais fortes, sendo exigida uma maior adaptação ao estresse hídrico e às altas temperaturas (MORAES et al., 2009).

Os inselbergues desempenham um papel importante na preservação da biodiversidade e formação de refúgios para as espécies que não conseguem crescer na planície circundante (BURKE, 2002; OLIVEIRA; GODOY, 2007; MORAES et al., 2009), justificando-se sua inclusão em estudos detalhados para a criação de áreas de conservação.

Embora sejam frequentes nas paisagens brasileiras, ainda são poucos os estudos florísticos e ecológicos desenvolvidos em inselbergues (OLIVEIRA; GODOY, 2007; ESGARIO et al., 2009), inclusive na região Nordeste (FEVEREIRO; FEVEREIRO, 1980; FRANÇA et al., 1997; ROCHA; AGRA, 2002; FRANÇA et al., 2006; ALMEIDA et al., 2007a; 2007b; CONCEIÇÃO et al., 2007; NEVES; CONCEIÇÃO, 2007; ARAÚJO et al., 2008; PORTO et al., 2008; GOMES; ALVES, 2009; MORAES et al., 2009; FABRICANTE et al., 2010) e, em especial no estado da Paraíba (FEVEREIRO; FEVEREIRO, 1980; ROCHA; AGRA, 2002; ALMEIDA et al., 2007a; 2007b; PORTO et al., 2008; FABRICANTE et al., 2010), sendo estes estudos de fundamental importância para a conservação dessas áreas. Assim, este trabalho realizou o levantamento florístico de um inselbergue no município de Puxinanã, Paraíba, Nordeste do Brasil, visando contribuir para o conhecimento da flora local e ampliar os estudos florísticos em inselbergues da região Nordeste, mais precisamente no semiárido da Paraíba.

\section{Material e Métodos}

\section{Área de estudo}

O presente estudo foi desenvolvido em um inselbergue localizado no município de Puxinanã (0708'62,1”S, 3558'31,4”W), Paraíba, Nordeste do Brasil (Figura 1). É uma rocha granítica, com aproximadamente $10 \mathrm{~m}$ de altura compreendendo uma área de $15.000 \mathrm{~m}^{2}$. Trabalhos de cunho florístico eram, até então, inexistentes para os inselbergues da região aspecto este, que, aliado às peculiaridades exibidas por esses ambientes rochosos (alta insolação e endemismo), culminaram a seleção dessa área para realização do estudo.

O município atinge, em sua cota máxima, $711 \mathrm{~m}$ de altitude e encontra-se situado na Mesorregião Agreste do estado da Paraíba. Sua área é de $74 \mathrm{~km}^{2}$ representando $0,1305 \%$ do estado e distando $121,2 \mathrm{~km}$ da capital. O relevo é geralmente movimentado, com vales profundos e estreitos dissecados (BELTRÃO et al., 2005), podendose observar um extenso agrupamento de inselbergues assentados nos espaços urbano e rural.

O Agreste paraibano caracteriza-se como área de transição entre a zona da mata e a zona das caatingas interioranas, com trechos quase tão úmidos quanto o litoral e outros secos como o sertão (PEREIRA et al., 
2002). Por esta razão, o clima da cidade é tropical com a estação chuvosa iniciando entre os meses de janeiro/ março e terminando entre os meses de julho/agosto (AESA, 2006). Ainda de acordo com a AESA (2006), os índices pluviométricos observados na sede do município registram uma média histórica de $651,0 \mathrm{~mm} / \mathrm{ano}$, a temperatura máxima registrada foi de $28^{\circ} \mathrm{C}$ e mínima de $16^{\circ} \mathrm{C}$.

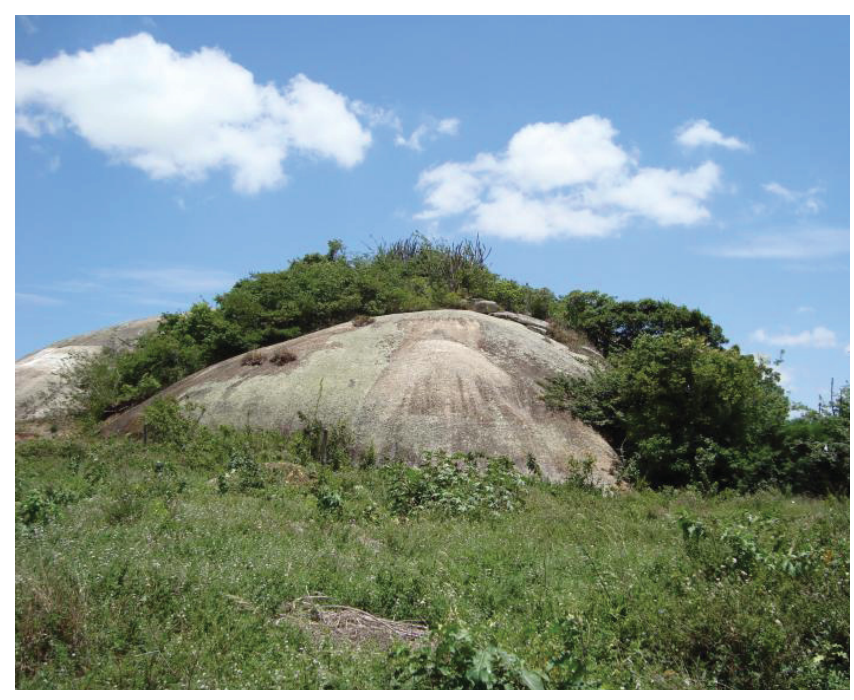

FIGURA 1: Vista parcial da área de estudo

(Foto: A. R. L. Pereira).

A fitofisionomia predominante é a Caatinga arbustiva (FERNANDES, 2000), com vegetação rala atingindo de 1,5 a $3 \mathrm{~m}$ de altura e presença de afloramentos rochosos. Observou-se que a área tem sofrido com a ação antrópica e a vegetação localizada no entorno do inselbergue estudado já foi quase totalmente devastada.

\section{Florística}

Foram realizadas coletas quinzenais aleatórias em trechos compreendendo a maior parte da área do inselbergue entre os meses de fevereiro e outubro de 2010. Os espécimes obtidos em campo foram prensados e herborizados e, em seguida, incorporados à coleção do Herbário Manuel de Arruda Câmara (ACAM) da Universidade Estadual da Paraíba (UEPB), Campus I, Campina Grande, Paraíba, Brasil.

As identificações fundamentaram-se na literatura especializada, consultas a especialistas ou, ainda, através de comparações com materiais depositados nos herbários: EAN, JPB, UEC e SPF (listados de acordo com HOLMGREN et al. 2006). A lista florística foi organizada em ordem alfabética e a proposta de classificação adotada foi a do APG II (2003). A grafia dos nomes científicos e autores das espécies foram consultados na base de dados do Missouri Botanical Garden ( $\mathrm{W}^{3}$ TROPICOS, 2010) e na Lista de Espécies da Flora do Brasil (FORZZA et al., 2010). O hábito foi definido considerando-se os dados observados em campo, bem como o descrito na literatura para a espécie seguindo a denominação do IBGE (1992).

\section{Resultados}

Foram coletadas 97 espécies pertencentes a 35 famílias, sendo 83 identificadas no nível de espécie, três no de gênero e 10 no nível de família. As espécies identificadas englobam 34 famílias e 69 gêneros (Tabela 1). As cinco famílias melhor representadas em número de espécies foram: Asteraceae (12 spp.), Euphorbiaceae e Fabaceae (nove spp. cada), Rubiaceae e Convolvulaceae (oito spp. cada), compreendendo $47,42 \%$ do total de espécies registradas (Figura 2).

Com relação ao número de gêneros, as famílias que se destacaram foram Fabaceae, com oito; Euphorbiaceae; com sete; Asteraceae, com seis e Rubiaceae, com cinco. Os gêneros mais representativos na área foram Ipomoea L. - Convolvulaceae, com cinco espécies, e Croton L. - Euphorbiaceae, com três espécies. A flora da área mostrou-se predominantemente herbáceo-arbustiva (61\%), embora com uma presença bastante destacada de trepadeiras (20\%) (Figura 3).

As famílias que se sobressaíram em número de representantes herbáceo-arbustivos foram: Asteraceae, com nove; Malpighiaceae e Rubiaceae, com quatro cada, seguidas de Boraginaceae e Commelinaceae, com três espécies cada. Dentre as trepadeiras, o segundo tipo de hábito mais representativo, as famílias mais diversas foram Convolvulaceae, com oito espécies, seguida de Euphorbiaceae e Vitaceae, com duas espécies cada. 
TABELA 1: Lista das espécies do inselbergue estudado no município de Puxinanã (0708'62,1"S - 3558'31,4”W), Paraíba, Nordeste do Brasil. Hábito: TRE $=$ Trepadeira; ARB = Arbusto; ARV = Árvore; EPI = Epífita; ERV = Erva; SUB = Subarbusto; RUP = Rupícola.

\begin{tabular}{|c|c|c|}
\hline FAMILÍA/ESPÉCIE & HÁBITO & VOUCHER \\
\hline \multicolumn{3}{|l|}{ ALSTROEMERIACEAE } \\
\hline Bomarea edulis (Tussac.) Herb. & ERV & Pereira 22 (ACAM) \\
\hline \multicolumn{3}{|l|}{ AMARANTHACEAE } \\
\hline Gomphrena vaga Mart. & ERV & Tölke 35 (ACAM) \\
\hline \multicolumn{3}{|l|}{ APOCYNACEAE } \\
\hline Mandevilla tenuifolia (J.C. Mikan) Woodson & ERV & Tölke 01 (ACAM) \\
\hline \multicolumn{3}{|l|}{ ARISTOLOCHIACEAE } \\
\hline Aristolochia birostris Duch. & TRE & Silva 21 (ACAM) \\
\hline \multicolumn{3}{|l|}{ ASTERACEAE } \\
\hline Acanthospermum hispidum DC. & ERV & Pereira 23 (ACAM) \\
\hline Bidens pilosa L. & ERV & Silva 28 (ACAM) \\
\hline Centratherum punctatum Cass. & ERV & Pereira 24 (ACAM) \\
\hline Conocliniopsis prasiifolia (DC.) R.M. King \& H. Rob. & $\mathrm{ARB}$ & Silva 23 (ACAM) \\
\hline Conyza bonariensis (L.) Cronquist & ERV & Silva 29 (ACAM) \\
\hline Galinsoga parviflora Cav. & ERV & Tölke 44 (ACAM) \\
\hline Lepidaploa chalybaea (Mart. ex DC.) H.Rob. & ERV & Silva 04 (ACAM) \\
\hline Pectis linifolia L. & ERV & Silva 09 (ACAM) \\
\hline Pithecoseris pacourinoides Mart. ex DC. & ERV & Tölke 45 (ACAM) \\
\hline Tridax procumbens L. & ERV & Silva 30 (ACAM) \\
\hline Asteraceae sp. 1 & ERV & Tölke 37 (ACAM) \\
\hline Asteraceae sp. 2 & ERV & Pereira 25 (ACAM) \\
\hline \multicolumn{3}{|l|}{ BEGONIACEAE } \\
\hline Begonia saxicola A. DC. (Figura 4-A) & RUP & Tölke 40 (ACAM) \\
\hline \multicolumn{3}{|l|}{ BIGNONIACEAE } \\
\hline Dolichandra unguis-cati (L.) L.G.Lohmann & $\mathrm{ARB}$ & Silva 01 (ACAM) \\
\hline \multicolumn{3}{|l|}{ BORAGINACEAE } \\
\hline Heliotropium angiospermum Murray & ERV & Tölke 05 (ACAM) \\
\hline Tournefortia salzmannii DC. & TRE & Pereira 09 (ACAM) \\
\hline Tournefortia rubicunda Salzm. ex A. DC. & TRE & Tölke 03 (ACAM) \\
\hline Varrronia dardani (Taroda) J.S. Mill. & $\mathrm{ARB}$ & Silva 07 (ACAM) \\
\hline \multicolumn{3}{|l|}{ BROMELIACEAE } \\
\hline Encholirium spectabile Mart. ex Schult. \& Schult.f. & RUP & Pereira 16 (ACAM) \\
\hline Tillandsia recurvata (L.) L. & EPI & Pereira 15 (ACAM) \\
\hline \multicolumn{3}{|l|}{ CACTACEAE } \\
\hline Melocactus ernestii Vaupel & RUP & Silva 33 (ACAM) \\
\hline Pilosocereus gounellei (F.A.C. Weber) Byles \& G.D. Rowley & RUP & Pereira 30 (ACAM) \\
\hline \multicolumn{3}{|l|}{ CAPPARACEAE } \\
\hline Capparis sp. & ERV & Pereira 26 (ACAM) \\
\hline \multicolumn{3}{|l|}{ COMMELINACEAE } \\
\hline Commelina obliqua Vahl & ERV & Silva 06 (ACAM) \\
\hline Dichorisandra hexandra (Aubl.) Kuntze ex Hand.-Mazz. (Figura 4-B) & ERV & Pereira 29 (ACAM) \\
\hline Tradescantia ambigua Mart. & ERV & Tölke 49 (ACAM) \\
\hline \multicolumn{3}{|l|}{ CONVOLVULACEAE } \\
\hline Ipomoea marcellia Meisn. & TRE & Pereira 21 (ACAM) \\
\hline Ipomoea nil (L.) Roth & TRE & Silva 32 (ACAM) \\
\hline
\end{tabular}


Ipomoea pes-caprae (L.) R. Br.

TRE Ipomoea phyllomega (Vell.) House

Ipomoea procurrens Meisn.

Jacquemontia evolvuloides (Moric.) Meisn.

Jacquemontia cf. multiflora (Choisy) Hallier f.

Merremia aegyptia (L.) Urb.

\section{CUCURBITACEAE}

Momordica charantia L.

\section{CYPERACEAE}

Cyperaceae sp.

\section{EUPHORBIACEAE}

Astraea lobata (L.) Klotzsch

Cnidoscolus aff. urens (L.) Arthur

Croton heliotropiifolius Kunth.

Croton lundianus (Didr.) Müll. Arg.

Croton rottlerifolius Baill.

Dalechampia brasiliensis Lam.

Euphorbia bahiensis (Klotzsch \& Garcke) Boiss.

Euphorbia comosa Vell.

Tragia volubilis L.

\section{FABACEAE}

Centrosema sagittatum (Humb. \& Bonpl. ex Willd.) Brandegee

Crotalaria bahiensis Windler \& S.G. Skinner (Figura 4-C)

Dioclea grandiflora Mart. ex Benth.

Mimosa paraibana Barneby

Poincianella pyramidalis (Tul.) L.P. Queiroz

Senegalia riparia (Kunth) Britton \& Rose ex Britton \& Killip

Senna macranthera (DC. ex Collad.) H.S. Irwin \& Barneby

Senna martiana (Benth.) H.S. Irwin \& Barneby

Zornia curvata Mohlenbr.

LAMIACEAE

Rhaphiodon echinus Schauer

MALPIGHIACEAE

Stigmaphyllon blanchetii C.E. Anderson

Malpighiaceae sp. 1

Malpighiaceae sp. 2

Malpighiaceae sp. 3

\section{MALVACEAE}

Sidastrum paniculatum (L.) Fryxell

Waltheria americana $\mathrm{L}$.

Sida galheirensis Ulbr.

MOLLUGINACEAE

Mollugo verticillata L.

\section{MYRTACEAE}

Myrtaceae sp. 1

Myrtaceae sp. 2

NYCTAGINACEAE

Boerhavia diffusa L.

\section{ORCHIDACEAE}

Brassavola tuberculata Hook. (Figura 4-D)
Tölke 41 (ACAM)

Tölke 38 (ACAM)

Tolke 42 (ACAM)

Tölke 50 (ACAM)

Silva 10 (ACAM)

Silva 19 (ACAM)

Pereira 19 (ACAM)

Pereira 07 (ACAM)

Tölke 24 (ACAM)

Tölke 21 (ACAM)

Tölke 27 (ACAM)

Pereira 14 (ACAM)

Tölke 23 (ACAM)

Silva 05 (ACAM)

Silva 02 (ACAM)

Pereira 05 (ACAM)

Tölke 31 (ACAM)

Tölke 25 (ACAM)

Tölke 36 (ACAM)

Silva 17 (ACAM)

28 (ACAM)

(ACAM)

Tölke 30 (ACAM)

Tölke 26 (ACAM)

Pereira 01 (ACAM) 
Cyrtopodium holstii L.C. Menezes

OXALIDACEAE

Oxalis divaricata Mart. ex Zucc.

PASSIFLORACEAE

Passiflora luetzelburgii Harms

\section{POACEAE}

Megathyrsus maximus (Jacq.) B.K.Simon \& S.W.L.Jacobs

Urochloa plantaginea (Link) R.D. Webster

PORTULACACEAE

Talinum triangulare (Jacq.) Willd.

RANUNCULACEAE

Clematis dioica $\mathrm{L}$.

RUBIACEAE

Guettarda sericea Müll. Arg.

Guettarda sp.

Manettia cordifolia Mart.

Richardia grandiflora (Cham. \& Schltdl.) Steud.

Mitracarpus sp.

Tocoyena formosa (Cham. \& Schltdl.) K. Schum.

Rubiaceae sp. 1

Rubiaceae sp. 2

SAPINDACEAE

Serjania glabrata Kunth

SOLANACEAE

Capsicum parvifolium Sendtn.

Solanum paniculatum L.

Solanum rhytidoandrum Sendtn.

\section{TURNERACEAE}

Turnera cearensis Urb.

Turnera subulata $\mathrm{Sm}$.

\section{VERBENACEAE}

Lantana camara L.

Lippia gracilis Schauer

\section{VITACEAE}

Cissus decidua Lombardi

Cissus erosa Rich.

INDETERMINADA
Pereira 12 (ACAM)

ERV

Pereira 10 (ACAM)

TRE

Silva 11 (ACAM)

ERV

ERV

Silva 24 (ACAM)

Silva 25 (ACAM)

ERV

Pereira 13 (ACAM)

TRE

Silva 13 (ACAM)

ARB

Silva 31 (ACAM)

ARB

TRE

ERV

Tölke 32 (ACAM)

Pereira 20 (ACAM)

ERV

Tölke 04 (ACAM)

Silva 22 (ACAM)

ARV Tölke 02 (ACAM)

ERV Tölke 39 (ACAM)

ERV Silva 18 (ACAM)

TRE

Tölke 43 (ACAM)

$\mathrm{ARB}$

Tölke 47 (ACAM)

$\mathrm{ARB}$

Pereira 11 (ACAM)

ARB

Pereira 12 (ACAM)

SUB Tölke 15 (ACAM)

TRE Silva 16 (ACAM)

ARB Pereira 27 (ACAM)

ARB Pereira 18 (ACAM)

TRE Pereira 28 (ACAM)

TRE Silva 15 (ACAM)

ARB Silva 34 (ACAM)
No que concerne ao nível de endemismo, verificouse que do total de espécies encontradas 10 delas são consideradas endêmicas à flora da Caatinga, a seguir listadas: Pectis linifolia L. e Pithecoseris pacourinoides Mart. ex DC. (Asteraceae), Varronia dardani (Taroda) J.S. Mill. (Boraginaceae), Encholirium spectabile Mart. ex Schult. \& Schult.f. (Bromeliaceae), Ipomoea marcellia Meisn. (Convolvulaceae), Crotalaria bahiensis Windler \& S.G. Skinner, Dioclea grandiflora Mart. ex Benth., Mimosa paraibana Barneby e Senna martiana (Benth.) H.S. Irwin \& Barneby (Fabaceae) e Guettarda sericea Müll.Arg. (Rubiaceae). Dentre essas espécies, Crotalaria bahiensis Windler \& S.G. Skinner, Ipomoea marcellia Meisn., Passiflora luetzelburgii Harms e Pectis linifolia L. foram, nesse estudo, referidas pela primeira vez para a flora paraibana. Tais dados apontam a necessidade de um programa intensivo de coletas nessas formações rochosas no estado da Paraíba e em outras áreas de caatinga do Nordeste do Brasil. 


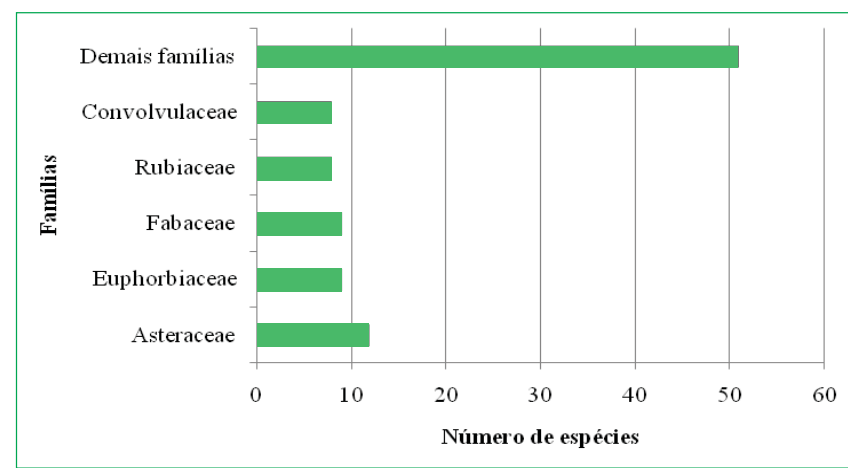

FIGURA 2: Famílias mais representativas em número de espécies amostradas em um inselbergue no município de Puxinanã, Paraíba, Nordeste do Brasil.
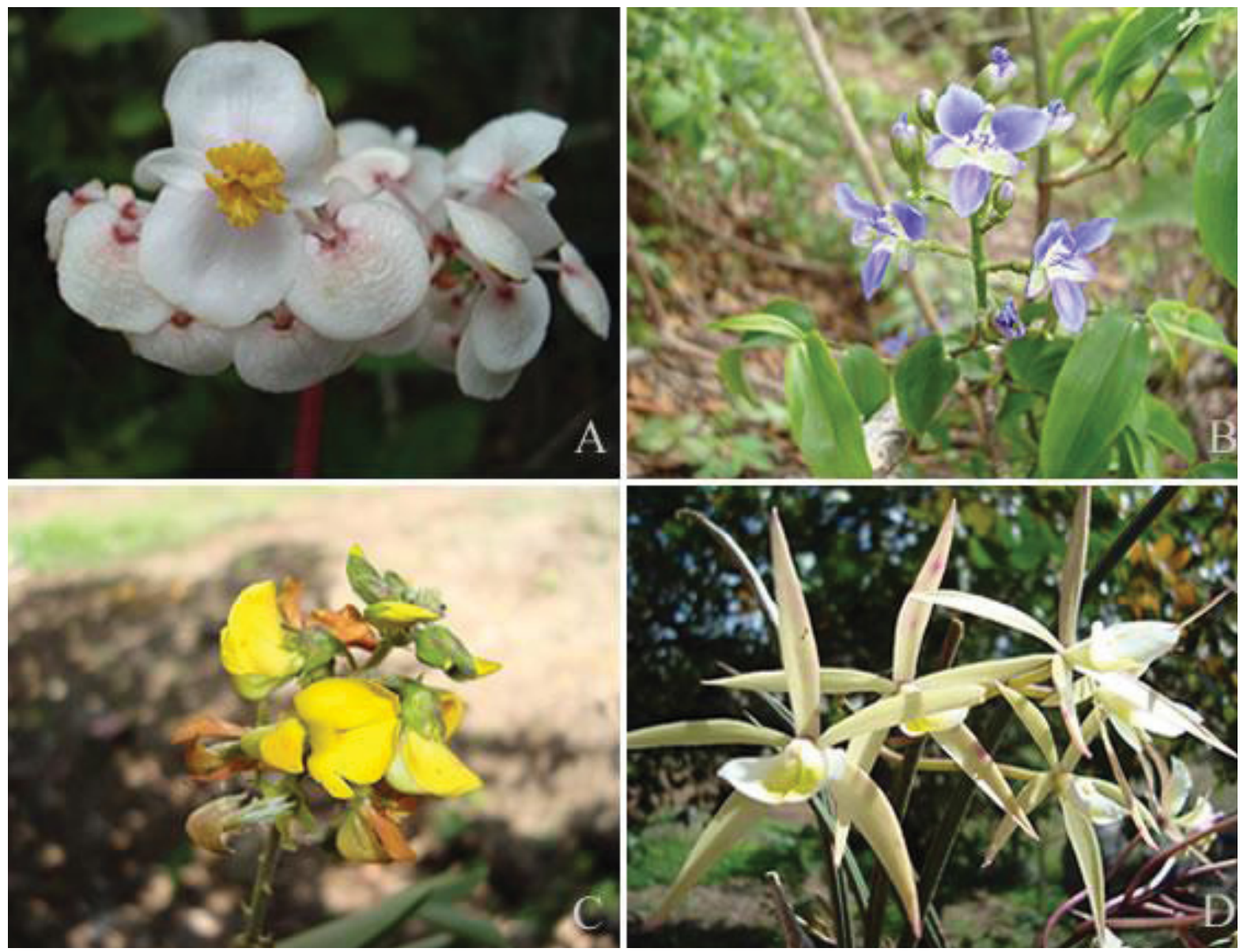

FIGURA 4: Alguns representantes da área de estudo. A. Begonia saxicola A. DC. (Begoniaceae). B. Dichorisandra hexandra (Aubl.) Kuntze ex Hand.-Mazz. (Commelinaceae). C. Crotalaria bahiensis Windler \& S.G. Skinner (Fabaceae). D. Brassavola tuberculata Hook. (Orchidaceae). (Fotos: A. R. L. Pereira).

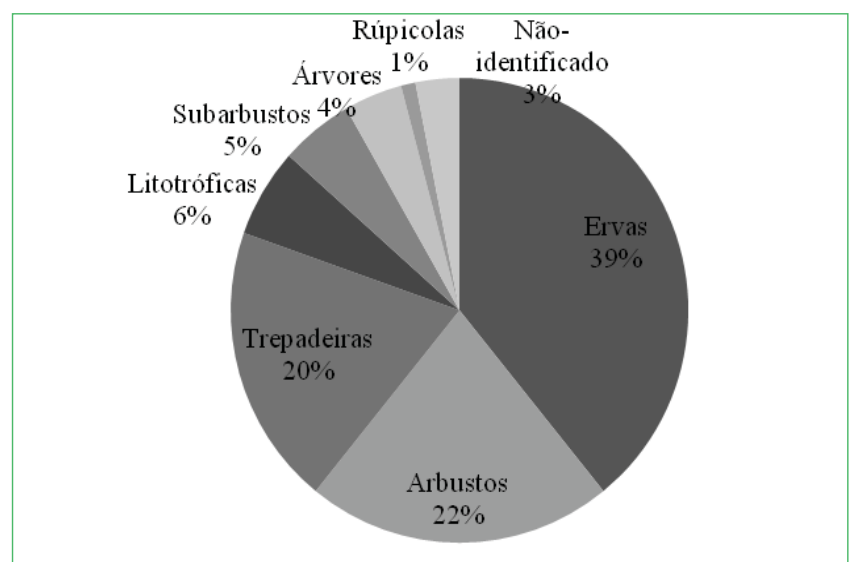

FIGURA 3: Percentual das espécies registradas em um inselbergue no município de Puxinanã, Paraíba, Nordeste do Brasil, segundo o hábito. 


\section{Discussão}

Segundo Barthlott et al. (1993) e Porembski (2007), as famílias Bromeliaceae, Cactaceae, Orchidaceae, Euphorbiaceae, Cyperaceae, Poaceae, Commelinaceae e Fabaceae são características nas formações vegetacionais dos inselbergues da América do Sul. Os autores ainda destacam os gêneros Cyrtopodium R. Br. (Orchidaceae), Melocactus Link \& Otto (Cactaceae) e Tillandsia L. (Bromeliaceae). Este inventário revelou a ocorrência de 31 espécies das famílias supracitadas e três espécies pertencentes aos gêneros citados nos trabalhos de Barthlott et al. (1993) e Porembski (2007), sendo suas representantes consideradas resistentes à dessecação e algumas delas inclusive suculentas, o que favorece a colonização sobre as rochas submetidas às condições ambientais peculiares a esse ambiente (POREMBSKI et al., 1997; POREMBSKI, 2007).

Poaceae e Cyperaceae compreendem as famílias relatadas entre as que ocorrem em inselbergues em todo o mundo (POREMBSKI, 2007), estando ambas representadas na área de estudo. Este mesmo autor refere Poaceae e Cyperaceae como às famílias mais comuns na base de inselbergues, aspecto também corroborado pelo presente trabalho. As famílias Euphorbiaceae, Bromeliaceae, Fabaceae, Rubiaceae, Cactaceae, Bignoniaceae, Boraginaceae, Begoniaceae (Figura 4-A), Commelinaceae (Figura 4-B), Cyperaceae, Malpighiaceae, Malvaceae, Myrtaceae, Oxalidaceae, Portulacaceae, Convolvulaceae, Apocynaceae, Orchidaceae, Poaceae, Asteraceae, Sapindaceae e Solanaceae são citadas também com significativa representatividade na maioria dos trabalhos sobre inselbergues desenvolvidos em áreas de Caatinga (FEVEREIRO; FEVEREIRO, 1980; FRANÇA et al., 1997; 2006; ROCHA; AGRA, 2002; ALMEIDA et al., 2007b; CONCEIÇÃO et al., 2007; ARAÚJO et al., 2008; PORTO et al., 2008; MORAES et al., 2009).

Boraginaceae, Begoniaceae, Commelinaceae, Cyperaceae, Malpighiaceae, Malvaceae, Myrtaceae, Oxalidaceae, Portulacaceae, Convolvulaceae, Euphorbiaceae, Bromeliaceae, Fabaceae (Figura 4-C), Cactaceae, Bignoniaceae, Apocynaceae, Orchidaceae e Asteraceae são relatadas em trabalhos similares realizados no estado da Paraíba (FEVEREIRO; FEVEREIRO, 1980; ROCHA; AGRA, 2002; ALMEIDA et al., 2007a; 2007b; PORTO et al., 2008; FABRICANTE et al., 2010). As exceções seriam as famílias Velloziaceae, Lentibulariaceae, Eriocaulaceae, Xyridaceae, Gesneriaceae e Melastomataceae com nenhuma representatividade para a área estudada, o que contrasta com outros levantamentos realizados na América do Sul, especialmente para o Brasil (BARTHLOTT et al., 1993; POREMBSKI et al., 1997; MEIRELLES et al., 1999; CONCEIÇÃO et al., 2007; NEVES; CONCEIÇÃO, 2007; POREMBSKI, 2007). Nesse sentido, Porembski (2007), observa, que, fatores mesológicos como clima, tipo de solo e ou substrato, precipitação e ação antrópica podem influenciar diretamente de modo a favorecer ou não o estabelecimento e colonização de espécies em determinadas áreas.

Euphorbiaceae é mencionada em diferentes trabalhos como uma das famílias melhor representadas em afloramentos rochosos na América do Sul, sendo encontrada, principalmente, em áreas de Caatinga, dados estes confirmados no presente trabalho e nos estudos realizados por Barthlott et al. (1993), França et al. (1997; 2006), Porembski et al. (1997) e Gomes e Alves (2009). O gênero Croton foi representado por três espécies, e tem sido relatado como importante componente do estrato arbustivo de inselbergues associados à Caatinga (FRANÇA et al., 1997; GOMES; ALVES, 2009). Também nessa vegetação, Fabaceae é referida como uma das mais bem representadas em inselbergues (FRANÇA et al., 1997; NEVES; CONCEIÇÃO, 2007), bem como Asteraceae (NEVES; CONCEIÇÃO, 2007), sendo esta a família que apresentou o maior número de espécies no presente estudo.

Bromeliaceae, Cactaceae e Orchidaceae sobressairam-se por serem as únicas a colonizarem as superfícies nuas da área estudada, sendo citadas por Meirelles et al. (1999) como pioneiras em afloramentos rochosos. Apenas Brassavola tuberculata Hook. (Orchidaceae) (Figura 4-D) forma um extenso tapete sobre as rochas, constituído por expressivo número de indivíduos na área estudada, e as espécies de Cactaceae e Bromeliaceae, bem como Cyrtopodium holstii L.C. Menezes ocorreram em áreas isoladas. Porembski 
(2007) discorre sobre a presença de espécies de xerófitas crescendo sobre a superfície nua da rocha, a exemplo do gênero Cyrtopodium, representado neste levantamento pela espécie $C$. holstii L.C. Menezes, além de Brassavola tuberculata Hook, ambas pertencentes à família Orchidaceae, e de espécies de Bromeliaceae.

As monocotiledôneas encontram-se representadas pelas famílias Orchidaceae, Alstroemeriaceae, Commelinaceae, Poaceae, Cyperaceae e Bromeliaceae, com um total de $11,34 \%$ de todas as espécies levantadas. Barthlott et al. (1993) e Porembski et al. (1997) já haviam descrito a ocorrência generalizada de monocotiledôneas em afloramentos rochosos de regiões tropicais. Nesse contexto, Gomes e Alves (2009), Conceição et al. (2007) e Meirelles et al. (1999) destacaram que o padrão geral, em termos de famílias predominantes, é de domínio de monocotiledôneas. Apesar de não estarem bem representadas em número de espécies, constituem geralmente grandes populações na área estudada, o que confirma as observações dos autores supracitados.

O hábito herbáceo e arbustivo foi o predominante no inselbergue. No entanto, de acordo com Schnitzer (2005) as trepadeiras apresentam maior tolerância ao estresse hídrico em relação aos estratos epifítico, herbáceo, arbóreo e arbustivo, apresentando maior crescimento durante a estação seca, o que justifica o elevado grau de ocorrência das mesmas na área estudada, representando $20 \%$ do componente da vegetação.

Evidenciou-se, assim, que o inselbergue estudado constitui uma comunidade florística semelhante a outras neste tipo de formação no âmbito da Caatinga, permitindo o estabelecimento de representantes de diversas famílias, culminando na manutenção de expressiva diversidade da flora local.

A diversidade associada ao papel de refúgio de espécies xerófitas e de centros de endemismos, definido para os inselbergues neotropicais por Porembski e Barthlott (2000), configura-se como forte e plausível justificativa para implementação de estratégias conservacionistas para os inselbergues sul-americanos. Desse modo, percebe-se nitidamente que o inselbergue estudado trata-se de um ambiente de vital importância biológica regional, com a necessidade de execução de estudos, enfocando diferentes aspectos (fitossociologia, biologia reprodutiva, fenologia, síndromes florais e de dispersão, dentre outras abordagens). Estes estudos contribuirão para a conservação dessas formações rochosas na região Nordeste, sobremaneira, no domínio semiárido brasileiro.

\section{Agradecimentos}

Os autores agradecem a Rubens Teixeira Queiroz e a Jorge Yoshio Tamashiro, da Universidade Estadual de Campinas (UNICAMP), pela identificação e ou confirmação das espécies de Commelinaceae, Euphorbiaceae e Fabaceae; a Gerleni Lopes Esteves, do Instituto de Botânica de São Paulo (IBt), pela identificação das espécies de Malvaceae; a Daniela Cristina Zappi, do Royal Botanic Gardens (Kew), pela identificação das espécies de Cactaceae e a Jefferson Maciel, do Jardim Botânico de Recife, pela identificação das espécies de Poaceae. A Juliana Castelo Branco Brasileiro e a Juliana Sales Rodrigues da Universidade Estadual da Paraíba (UEPB), Campus I, em Campina Grande pelo auxílio durante os trabalhos de campo; e a Allysson Allan de Farias da Universidade Federal de Pernambuco (UFPE) pela obtenção das coordenadas do local de coleta.

\section{Referências}

AESA - AGÊNCIA EXECUTIVA DE GESTÃO DAS ÁGUAS DO ESTADO DA PARAÍBA. Boletim de informações climáticas. 2006. Disponível em: <http://www.aesa.pb.gov.br>. Acesso em: 6 set. 2010.

ALMEIDA, A.; FELIX, W. J. P.; ANDRADE, L. A.; FELIX, L. P. A família Orchidaceae em inselbergues da Paraíba, Nordeste do Brasil. Revista Brasileira de Biociências, Porto Alegre, v. 5, p. 753-755, 2007a.

ALMEIDA, A.; FELIX, W. J. P.; ANDRADE, L. A.; FELIX, L. P. Leguminosae na flora de inselbergs no estado da Paraíba, Nordeste do Brasil. Revista Brasileira de Biociências, Porto Alegre, v. 5, p. 750-752, $2007 \mathrm{~b}$.

APG II. An update of the Angiosperm Phylogeny Group classification for the orders and families of flowering plants: APG II. Botanical Journal of the Linnean Society, London, v. 141, p. 399-436, 2003.

ARAÚJO, F. S.; OLIVEIRA, R. F.; LIMA-VERDE, L. W. Composição, espectro biológico e síndromes de dispersão da vegetação de um inselberg no domínio da Caatinga, Ceará. Rodriguésia, Rio de Janeiro, v. 59, p. 659-671, 2008. 
BARTHLOTT, W.; GRÖGER, A.; POREMBSKI, S. Some remarks on the vegetation of tropical Inselbergs: diversity and ecological differentiation. Biogéographica, Paris, v. 69, p. 105-124, 1993.

BELTRÃO, B. A.; MORAIS, F.; MASCARENHAS, J. C.; MIRANDA, J. L. F.; JUNIOR, L. C. S.; MENDES, V. A. Projeto cadastro de fontes de abastecimento por água subterrânea: diagnóstico do município de Puxinanã, estado da Paraíba. Recife: CPRM/PRODEEM, 2005. 10 p.

BURKE, A. Island-matrix relationships in Nama Karoo inselberg landscapes. Part I: Do inselbergs provide a refuge for matrix species? Plant Ecology, Perth, v. 160, p. 79-90, 2002.

CONCEIÇÃO, A. A.; PIRANI, J. R.; MEIRELLES, S. T. Floristics, structure and soil of insular vegetation in four quartzite-sandstone outcrops of "Chapada Diamantina", Northeast Brazil. Revista Brasileira de Botânica, São Paulo, v. 30, p. 641-656, 2007.

ESGARIO, C. P.; FONTANA, A. P.; SILVA, A. G. A flora vascular sobre rocha no Alto Misterioso, uma área prioritária para a conservação da Mata Atlântica no Espírito Santo, Sudeste do Brasil. Natureza On Line, Santa Teresa, v. 7, p. 80-91, 2009.

FABRICANTE, J. R.; ANDRADE, L. A.; MARQUES, F. J. Caracterização populacional de Melocactus zehntneri (Britton \& Rose) Luetzelburg (Cactaceae) ocorrente em um inselbergue da Caatinga paraibana. Biotemas, Florianópolis, v. 23, p. 61-67, 2010. FERNANDES, A. Fitogeografia brasileira. 2. ed. Fortaleza: Multigraf, 2000. 341 p.

FEVEREIRO, P. C. A.; FEVEREIRO, V. P. B. Composição florística de alguns inselbergs do Estado da Paraíba: I - A flora da Pedra dos Caboclos: observações preliminares. Agropecuária Técnica, Areia, v. 1, p. 126-131, 1980.

FORZZA, R. C.; LEITMAN, P. M.; COSTA, A. F.; CARVALHO JR., A. A.; PEIXOTO, A. L.; WALTER, B. M. T.; BICUDO, C.; ZAPPI, D.; COSTA, D. P.; LLERAS, E.; MARTINELLI, G.; LIMA, H. C.; PRADO, J.; STEHMANN, J. R.; BAUMGRATZ, J. F. A.; PIRANI, J. R.; SYLVESTRE, L.; MAIA, L. C.; LOHMANN, L. G.; QUEIROZ, L. P.; SILVEIRA, M.; COELHO, M. N.; MAMEDE, M. C.; BASTOS, M. N. C.; MORIM, M. P.; BARBOSA, M. R.; MENEZES, M.; HOPKINS, M.; SECCO, R.; CAVALCANTI, T. B.; SOUZA, V. C. Lista de espécies da flora do Brasil. Rio de Janeiro: Jardim Botânico do Rio de Janeiro, 2010. Disponível em: $<$ http://floradobrasil.jbrj.gov.br/2010/FB006316>. Acesso em: 22 jul. 2011.

FRANÇA, F.; MELO, E.; GONÇALVES, J. M. Aspectos da diversidade da vegetação no topo de um inselberg no semi-árido da Bahia, Brasil. Sitientibus, Feira de Santana, v. 6, p.30-35, 2006.

FRANÇA, F.; MELO, E.; SANTOS, C. C. Flora de inselbergs da região de Milagres, Bahia, Brasil: I - Caracterização da vegetação e lista de espécies de dois inselbergs. Sitientibus, Feira de Santana, v. 17, p. 163-184, 1997.

GOMES, P.; ALVES, M. Floristic and vegetation aspects of an inselberg in the semi-arid region of northeast Brazil. Edinburgh Journal of Botany, Edinburgh, v. 66, p. 329-346, 2009.
HOLMGREN, P. K.; HOLMGREN, N. H.; BARNETT, L. C. 2006. Index Herbariorum - Part I - The herbaria of the world. Disponível em $\quad<$ http://sci.web.nybg.org/science2/IndexHerbariorum.as.> Acesso em: 10 nov. 2010.

IBGE - INSTITUTO BRASILEIRO DE GEOGRAFIA E ESTATÍSTICA. Manual técnico da vegetação brasileira. Série manuais técnicos em Geociências N1. Rio de Janeiro: IBGE, 1992. $89 \mathrm{p}$.

MEIRELLES, S. T.; PIVELLO, V. R.; JOLY, C. A. The vegetation of granite rock outcrops in Rio de Janeiro, Brazil, and the need for its protection. Environmental Conservation, Cambridge, v. 26, p. 10-20, 1999.

MORAES, A. O.; MELO, E.; AGRA, M. F.; FRANÇA, F. A família Solanaceae nos "Inselbergs" do semi-árido da Bahia, Brasil. Iheringia, Porto Alegre, v. 64, p. 109-122, 2009.

NEVES, S. P. S; CONCEIÇÃO, A. A. Vegetação em afloramentos rochosos na Serra do Sincorá, Chapada Diamantina, Bahia, Brasil. Sitientibus, Série Ciências Biológicas, Feira de Santana, v. 7, p. 36-45, 2007.

OLIVEIRA, R. B.; GODOY, S. A. P. Composição florística dos afloramentos rochosos do Morro do Forno, Altinópolis, São Paulo. Biota Neotropica, Campinas, v. 7, p. 37-47, 2007.

PEREIRA, O. M.; ANDRADE, L. A.; BARBOSA, M. R. V.; SAMPAIO, E. V. S. B. Composição florística e análise fitossociológica do componente arbustivo-arbóreo de um remanescente florestal no agreste paraibano. Acta Botanica Brasilica, São Paulo, v. 16, p. 357-369, 2002.

POREMBSKI, S. Tropical inselbergs: habitat types, adaptative strategies and diversity patterns. Revista Brasileira de Botânica, São Paulo, v. 20, p. 579-586, 2007.

POREMBSKI, S.; BARTHLOTT, W. Inselbergs: biotic diversity of isolated rock outcrops in tropical and temperate regions. Berlin: Springer-Verlag, 2000. $524 \mathrm{p}$.

POREMBSKI, S.; REINE, R.; BARTHLOTT, W. Inselberg vegetation and the biodiversity of granite outcrops. Journal of the Royal Society of Western Australia, Welshpool, v. 80, p. 193-199, 1997.

PORTO, P. A. F.; ALMEIDA, A.; PESSOA, W. J.; TROVÃO, D.; FELIX, L. P. Composição florística de um inselbergue no agreste paraibano, município de Esperança, Nordeste do Brasil. Revista Caatinga, Mossoró, v. 21, p. 214-222, 2008.

ROCHA, E. A.; AGRA, M. F. Flora do Pico do Jabre, Paraíba, Brasil: Cactaceae Juss. Acta Botanica Brasilica, São Paulo, v. 16, p. 15-21, 2002.

SCHNITZER, S. A. A mechanistic explanation for global patterns of liana abundance and distribution. The American Naturalist, Chicago, v. 166, p. 262-276, 2005.

$\mathrm{W}^{3}$ TROPICOS. Tropicos Home - Missouri Botanical Garden. 2010. Disponível em: <http: //www.tropicos.org/>. Acesso em: 5 set. 2010. 